\title{
Cryoneurolysis for the management of chronic pain in patients with knee osteoarthritis; a double-blinded randomized controlled sham trial
}

Niels-Peter Brøchner Nygaard ${ }^{1,2^{*}}$ (D), Carsten Koch-Jensen ${ }^{3}$, Henrik Bjarke Vægter ${ }^{4,5}$, Niels Wedderkopp ${ }^{6}$, Morten Blichfeldt-Eckhardt ${ }^{4,5}$ and Bibi Gram ${ }^{1,2}$

\begin{abstract}
Objective: Pain is the principal symptom in knee osteoarthritis $(\mathrm{OA})$. Current non-operative treatment options have only moderate effects and often patients experience persistent pain or side-effects. Novel advances in the field of cryoneurolysis applies low temperatures to disrupt nerve signaling at the painful area, providing pain relief. The primary aim of this randomized controlled trial $(\mathrm{RCT})$ is to investigate if cryoneurolysis is superior to sham at decreasing pain intensity 2 weeks after the intervention in patients with knee OA. Secondary aims are to explore effects on pain, quality of life and functional performance over 24 months.

Methods: This two-arm, parallel-group RCT, approved by the Regional Ethics Committee, will randomly allocate patients $(n=94)$ to a cryoneurolysis intervention group + standardized education and exercise $(C R Y O)$ or a sham group + standardized education and exercise (SHAM) (1:1 ratio). Both groups will be assessed at baseline, 2 weeks post intervention, post education and exercise and at 6,12 and 24 months after cryoneurolysis. The primary outcome is the NRS knee pain intensity score assessed 2 weeks post the intervention. Secondary outcome measures include functional performance (chair-stand test, $40 \mathrm{~m}$ walk, stair test and maximum voluntary contraction of the knee), patient reported outcomes (quality of life (EQ5D), Knee and osteoarthritis outcome scores (KOOS), among others), use of analgesics, and adverse events over 24 months.
\end{abstract}

Impact statement: Cryoneurolysis could potentially provide an effective, safe and non-pharmacological therapeutic option to treat pain in OA patients. The potential benefits include increased functional capacity and quality of life as a result of significant pain relief and improved benefits of physical exercise.

Trial registration: Clinicaltrials.gov, NCT03774121, registered 3 March 2018, http://www.clinicaltrials.gov

Keywords: Cryoneurolysis, Osteoarthrities, Knee pain, Exercise

\footnotetext{
* Correspondence: Niels-peter.brochner.nygaard@rsyd.dk

${ }^{1}$ Research Unit of Health Science, Hospital of South West Jutland, University Hospital of Southern Denmark, Esbjerg, Denmark

${ }^{2}$ Department of Regional Health Research, University of Southern Denmark, Odense, Denmark

Full list of author information is available at the end of the article
}

(c) The Author(s). 2021 Open Access This article is licensed under a Creative Commons Attribution 4.0 International License, which permits use, sharing, adaptation, distribution and reproduction in any medium or format, as long as you give appropriate credit to the original author(s) and the source, provide a link to the Creative Commons licence, and indicate if changes were made. The images or other third party material in this article are included in the article's Creative Commons licence, unless indicated otherwise in a credit line to the material. If material is not included in the article's Creative Commons licence and your intended use is not permitted by statutory regulation or exceeds the permitted use, you will need to obtain permission directly from the copyright holder. To view a copy of this licence, visit http://creativecommons.org/licenses/by/4.0/. The Creative Commons Public Domain Dedication waiver (http://creativecommons.org/publicdomain/zero/1.0/) applies to the data made available in this article, unless otherwise stated in a credit line to the data. 


\section{Background}

Chronic pain represents a major challenge worldwide, with significant clinical, social and economic implications. In Denmark and the rest of Europe, 20\% of all chronic pain conditions is related to osteoarthritis (OA) [1]. There are over 300.000 people diagnosed with OA per year in Denmark alone, and the incidence of this pathology will increase significantly [2]. The pain and loss of function associated with OA, results in a considerable amount of years lived with disability [3] and has significant socioeconomic consequences, estimated at 1 $2.5 \%$ of the gross domestic product in western countries [4]. Knee OA in particular, has a high prevalence rate compared to other types of OA, and is also present in the younger working age population [5].

The treatment of knee OA typically focuses on pain relief, however, the effects of current conservative treatment options remain small to moderate and most are associated with side effects [6]. In many cases, patients may be subjected to partial/total knee arthroplasty (TKA). TKA is considered to be an effective treatment for end-stage knee osteoarthritis [7], however, more than $20 \%$ of patients receiving TKA, experience persistent and unchanged pain post-surgery [8-10]. Therefore, effective and low-risk strategies are needed $[11,12]$. Cryoneurolysis, which is the application of low temperatures $\left[-20^{\circ} \mathrm{C}\right.$ to $\left.-100^{\circ} \mathrm{C}\right]$ to a target percutaneous peripheral nerve, causing Wallerian degeneration [13, 14], disrupts nerve function while structural elements of the nerve bundle remain intact. This allows for complete regeneration and functional recovery of the nerve over time [15, 16] and has shown promising short and long-term results in a variety of chronic pain conditions such as lumbar facet joint pain [17], plantar fasciitis [18], occipital neuralgia [19], post thoracotomy pain syndrome [20], and Morton's neuroma [21]. The ability to target the genicular nerves to reduce pain around the knee has been reported by studies applying radio frequency ablation (RF) [22-24] but cryoneurolysis has in that respect been associated with less adverse effects [25]. Radnovich et al. targeted the infrapatellar branch of the saphenous nerve (ISN) to reduce pain in patients with knee OA [26]. The authors reported significant pain relief for up to 150 days and no serious adverse effects. In another study, Dasa et al. introduced preoperative cryoneurolysis to the ISN and the anterior femoral cutaneous nerve (AFCN) prior to TKA in patients with knee OA and observed a statistically significant reduction in hospital stay (days), and a decrease in prescribed opioids [27]. These results provide clinical evidence, suggesting that cryoneurolysis treatment is a safe procedure, that may reduce both pain and symptoms in patients with knee OA $[14,28]$. Further adequately powered prospective randomized controlled trials (RCTs) are needed to confirm the efficacy and safety of cryoneurolysis treatment in patients with knee OA.

In Denmark, a standardized education and neuromuscular exercise program (GLA:D) has been implemented in the national clinical guidelines by the Danish Health Authority, for the treatment of knee and hip OA in clinical practice [29]. Recent studies show that exercise reduce pain and improve function in people with knee or hip OA [30, 31]. Despite of these reports, the beneficial effects remains moderate with difficulties in maintaining these effects at long-term follow-up [29]. In addition the implementation of the exercise program is not optimal [12] and includes a significant discontinuation rate for patients reporting high pain levels [29]. In this line, pain and muscle weakness, among others, have been reported to be major barriers for physical exercise [32, 33]. The application of cryoneurolysis treatment as an effective pain reducing treatment prior to GLA:D might provide significant pain relief and improve patients' ability to produce force, resulting in improved adherence, exercise effectiveness and long-term benefits [34]. Currently, no studies have reported the effects of cryoneurolysis treatment on pain and functional performance in conjunction with a standardized education and exercise programme (GLA:D), in patients with knee OA.

\section{Objective}

The primary objective of this RCT is to investigate if cryoneurolysis is superior to sham at decreasing pain intensity 2 weeks after the intervention in patients with painful knee OA.

The secondary objective is to explore the safety and effectiveness of cryoneurolysis followed by GLA:D and to assess long term effects.

\section{Methods}

This study protocol describes the design of an ongoing parallel-group RCT conducted at the Department of Neurology, University Hospital of Southern Denmark, Esbjerg, Denmark. The study protocol conforms to the Standard Protocol Items: Recommendations for Interventional Trials (SPIRIT) (see also appendix, Table 2), and The Consolidated Standards of Reporting Trials of Nonpharmacological Treatments (CONSORT NPT) will be used as a guideline for reporting this trial. The study is conducted according to the declaration of Helsinki and is approved by The Regional Committees on Health Research Ethics for Southern Denmark (S-20180089) and registered in ClinicalTrials.gov (NCT03774121).

\section{Participants and recruitment procedure}

This study is recruiting patients with pain and knee OA that are referred to GLA:D by their general practitioner, prior to assessment of surgery eligibility at the hospital (Fig. 1). 
Patients are screened according to the eligibility criteria outlined below, and the diagnosis is confirmed by further examination by an orthopaedic surgeon confirming knee $\mathrm{OA}$ and $\mathrm{OA}$ related pain. Eligible patients are invited to proceed to a second visit where nerves around the knee are identified using ultrasound (US) and electrical nerve stimulation followed by a diagnostic genicular nerve block. If patients experience a $\geq 50 \%$ decrease in knee pain intensity (NRS) as a result of the nerve block, they will be randomly allocated to either a cryoneurolysis intervention group (CRYO) or a sham group (SHAM).

\section{Inclusion criteria}

- Referred to GLA:D [29] by a physician.

- Age $\geq 18$ years

- Chronic knee pain for a minimum duration of 6 months.

- Pain intensity $\geq 4$ on the Numeric Rating Scale (NRS).

- Radiographic confirmation of osteoarthritis; Grade 2-4 changes according to the Kellgren-Lawrence classification system.

- A decrease of $\geq 50 \%$ in NRS scores with diagnostic genicular nerve block.

- Written and oral understanding of Danish.

\section{Exclusion criteria}

- History of systemic inflammatory conditions such as rheumatoid arthritis.

- Previous recipient of cryoneurolysis for the knee.

- Use of hyaluronic acid within the previous 30 days.

- Injection of corticosteroid within the previous 3 months.

- Clinically significant structural abnormities affecting locomotion and knee function aside from osteoarthritis and which might cause chronic knee pain.

- Body mass index $\leq 18$ and $\geq 40 \mathrm{~kg} / \mathrm{m} 2$.

- In treatment for other pain conditions.

- Pregnancy

- Coagulopathy

- Uncontrolled serious disease (cancer, diabetes, etc.)

- Disease associated with reactions to cold, such as cryoglobulinemia, cold urticarial and Renaud's syndrome.

\section{Randomization}

Randomization is performed as computer-generated block randomization with a 1:1 allocation ratio using random block sizes of 2,4 and 6 . The randomization restrictions will not be disclosed to ensure allocation concealment and the sequence will be performed by an external co-investigator. To account for the placebo effect and reduce the risk of bias, the patients, therapists and data-manager will be blinded to the allocation. Blinding will be assured using a sham trial that includes the same procedures as cryoneurolysis treatment but without any freezing temperatures.

The allocation code is concealed in a sealed envelope, that will be available for the surgeon performing the cryoneurolysis procedure only. In case of unexpected issues and if deemed absolutely necessary by the investigators and physician, unblinding will occur according to emergency unblinding procedures that will maintain the integrity and confidentiality of the study.

\section{Interventions}

Active and sham cryoneurolysis

The interventions involve two visits.

First visit Radiographic confirmation and clinical determination of knee OA as pain generator followed by the identification of the infrapatellar branch of the saphenous nerve (IBSN) and anterior femoral cutaneous nerve (AFCN). Predefined areas for each nerve are marked directly on the patient $[35,36]$. The area is searched using a transcutaneous electrical nerve stimulation (TENS) wand. Areas where the patient reported a response are marked, and then retraced with incrementally lower current to further specify nerve location. The location is finally visually confirmed using ultra-sound. Nerve structures can be distinguished by its hypoechoic nerve fascicles among the hyperechoic epineurium forming a honeycomb-like structure in short-axis view. A diagnostic nerve block using Ropivacain $(5 \mathrm{mg} / \mathrm{ml})$ is performed at each location guided by ultra-sound. All patients reporting a decrease of $\geq 50 \%$ in NRS pain scores are scheduled for a second visit for cryoneurolysis.

Second visit Ropivacain $(5 \mathrm{mg} / \mathrm{ml})$ is injected before treatment, locally at the insertion-point, $4-6 \mathrm{~cm}$ from target nerve locations. This allows for continuous patient feedback during the following procedures. The cryoneurolysis probe (Iceseed 1.5, Galil Medical Ltd.) is inserted in proximity of the target nerve, guided by ultrasound visualization to accurately determine the location of the nerve and to account for adjacent neurovascular structures and variations in anatomical structures. Cryoneurolysis are performed with a single freeze cycle; $30 \mathrm{~s}$ at an effect of $20 \%$, and 2 min 30 s at $60 \%$ effect. After each freezing cycle, $1 \mathrm{~min}$ active thaw and $1 \mathrm{~min}$ passive thaw is used. The machine used for cryoneurolysis is a VisuaIICE, (Galil Medical Ltd.), which utilizes Argon as a coolant and Helium to thaw. This technology allows for reversible destruction of nerves, also known as Wallerian 
degeneration, that prevents nerve signaling and potentially alleviate pain and motor dysfunction in a number of medical conditions.

The sham intervention includes the same procedures as described above but using a sham probe that does not allow for any freezing temperatures. Thus, visible marks as a result of the procedures will be similar in both groups.

\section{GLA:D}

Following the cryoneurolysis intervention, both groups will participate in GLA:D [29] for a duration of 8 weeks provided by specialized physiotherapists. The GLA:D program consists of patient education and neuromuscular exercise. Patient education consists of three sessions over the course of 2 weeks given by trained physiotherapists and focus on giving the patient knowledge on osteoarthritis and treatment with exercise. Following patient education, patients participates in a 6-week groupbased NEuroMuscular Exercise program (NEMEX) with a total of 12 sessions, each session lasting for $60 \mathrm{~min}$ [31]. Patients who do not wish to or are not able to participate in the supervised exercise program, have the choice to perform the program at home, with detailed instructions by the physiotherapist. The NEMEX of the GLA:D program is performed in groups and with the supervision of an experienced physiotherapist specialized in training of musculoskeletal disorders.

\section{Outcomes}

The patients will be assessed at baseline, 2 weeks post intervention, after completion of GLA:D and 6, 12- and 24- months post cryoneurolysis. The tests will include both patient-reported outcomes (PRO) and objective functional performance tests. The effects of group CRYO and group SHAM will be compared at each timepoint (Table 1).

\section{Primary outcome}

The primary outcome is the change in patient reported knee pain intensity assessed using the numeric rating scale (NRS) from baseline to 2 weeks post cryoneurolysis compared between group CRYO and SHAM. NRS consists of an 11-point scale between 0 and 10-, anchored by two verbal descriptors, "no pain" for the score of 0 and "worst pain imaginable" for the score of 10. Respondents are asked to rate their pain intensity on average within the past $24 \mathrm{~h}$. The NRS instrument has been validated in diverse populations and has been widely applied in clinical and research settings [37].

\section{Secondary outcomes}

Functional performance will be evaluated by the $30 \mathrm{~s}$ chair-stand test, the $40 \mathrm{~m}$ fast-paced walk test, the 9-step stair-climb test and isometric knee MVC force [38].
The 30 s chair-stand test [39] consists of repeated sitto-stand movement for a duration of $30 \mathrm{~s}$. The starting position is seated, with feet placed flat on the floor, shoulder width apart and with the arms crossed on the chest. The position change to standing, with hips and knees fully extended, followed by sitting back down, with bottom fully touching the seat. The test is performed with usual footwear and the chair should be with a straight back, with no arms, placed against a wall. In cases where the movement cannot be performed even once, the hands are allowed to be placed on the legs or a regular mobility aid can be used - the result is then reported as an adapted test score. The outcome is the total number of complete chair stands performed for the duration of the task (one chair stand represents a stand followed by a sit movement).

The $40 \mathrm{~m}$ fast paced walk test [40] consists of walking as fast as possible, but still safely, along a $10 \mathrm{~m}$ marked walkway, then turning around a cone / tape and return. This is then repeated for a total distance of $40 \mathrm{~m}$. The test is performed with usual footwear and regular walking aid is allowed and recorded. The outcome is expressed as speed. i.e. walking distance $(40 \mathrm{~m})$ divided by the time to perform the task (s). Timing is paused during turns.

The 9-step stair-climb test [41] consists of the ascend and descend a flight of stairs as fast as possible, but still safely. The flight of stairs preferably has 9 steps, step height appx. $20 \mathrm{~cm}$, with handrails. The test is performed with usual footwear and regular walking aid is allowed and recorded. The outcome is the total time to perform the task (s).

Quadriceps strength will be assessed measuring isometric MVC force of the knee extensors (Bofors Elektronik, Karlskoga, Sweden). Patients will be seated in a chair with knee and hip flexed at $90^{\circ}$ and with the pelvis and chest restrained by straps. A non-extensile chain attached to the back of the chair and connected to a force transducer will be placed just proximal to the malleolus. The patient will then be asked to perform three knee extensions pushing as hard as possible against the chain, with $1 \mathrm{~min}$ rest in between. The highest peak value out of the three MVCs will be taken as the MVC force.

In addition to pain intensity, PRO-data will be collected using PainData, which is an electronic questionnaire system and database with multiple integrated questionnaires, including:

- Knee Injury and Osteoarthritis Outcome Score (KOOS). To assess patients' opinion about their knee and associated problems [42].

- EQ-5D. To assess generic quality of life [43].

- Pain Catastrophizing Scale. To assess the extent of catastrophic thinking [44]. 


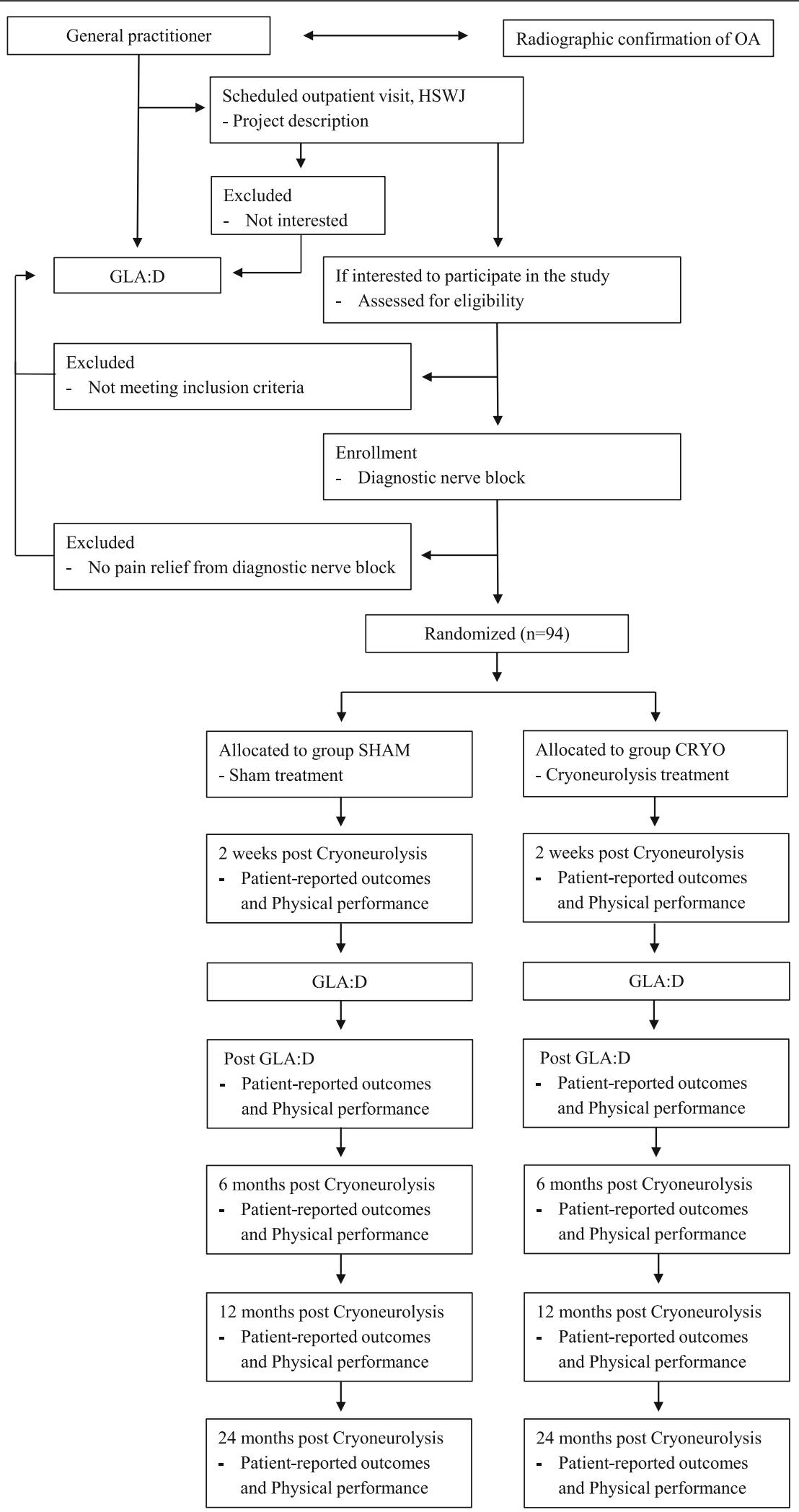

Fig. 1 Flow chart 
- Patient Health Questionaire (PHQ9). To assess the extent of depression [45].

- Generalized Anxiety Disorder (GAD7). To assess the extent of anxiety [46].

- Pain intensity (NRS) and location (electronic drawing on a $2 \mathrm{D}$ model).

- Self-reported use of analgesics.

Adverse events will also be reported and are defined as any undesirable experience during the trial leading to contact with the healthcare system (general practitioner, emergency room or hospital). All adverse effects will be assessed during follow-ups using both pre-specified symptom inventories and open-ended questions.

\section{Sample size}

The planned number of trial participants is based on the null-hypothesis, assuming no difference between cryoneurolysis treatment and sham. Estimating the sample size for a two-sample means test with a level of significance at 0.05 , assuming a common standard deviation (SD) of 3 in NRS pain intensity scores indicates that for the intention-to-treat (ITT) population, 74 individuals is required to obtain a power of at least $80 \%$ to establish a minimal clinically important difference (MCID) of 2 in NRS pain scores [47]. The MCID and common standard deviation is based on previous findings with a similar patient group and intervention [48]. With an expected drop-out rate of $20 \%$, a total of 94 individuals will be included in the project, 47 in each group.

\section{Statistical methods}

To evaluate the distributions of the continuous outcomes, visual inspection of the studentized residuals will be applied to evaluate whether the assumption of normality is reasonable. Data will be reported as differences between group means (means \pm standard deviations, 95\% CIs) if normal distributed, otherwise as medians with interquartile ranges. Categorical data will be reported as numbers and proportions. An Intention-To-Treat (ITT) analysis will be used for all allocated patients. Mixed linear regression with the assumption of unstructured covariance will be used to model the effect of the cryoneurolysis treatment over time and to take into account the repeated measures by including individuals as random variables. Time, group of treatment (cryoneurolysis or SHAM) and the Group, time interaction will be used as fixed effects to estimate different patterns of change over time. The results will be illustrated using marginal effects that will be calculated using the margins command of STATA16. If the 95\% CIs do not overlap, data will be considered statistically significant. A statistically significant difference of at least two in NRS scores between groups, will be interpreted as a MCID.

Table 1 Timeline of the study - outcomes and variables assessed during the trial period

\begin{tabular}{|c|c|c|c|c|c|c|c|c|c|}
\hline \multirow[t]{3}{*}{ Timepoint } & \multicolumn{9}{|c|}{ Study period } \\
\hline & \multicolumn{2}{|c|}{ Enrolment } & \multirow{2}{*}{$\begin{array}{l}\text { Allocation } \\
\text { Ow }\end{array}$} & \multicolumn{3}{|c|}{ Post-allocation } & \multicolumn{3}{|c|}{ Follow-up } \\
\hline & $-4 w$ & $-1 w$ & & $2 w$ & 10w & na & $24 w$ & $48 w$ & $96 w$ \\
\hline \multicolumn{10}{|l|}{ Enrolment: } \\
\hline Eligibility screening & $x$ & & & & & & & & \\
\hline Informed consent & $x$ & & & & & & & & \\
\hline Genicular nerve block & & $x$ & & & & & & & \\
\hline Allocation & & & $x$ & & & & & & \\
\hline \multicolumn{10}{|l|}{ Interventions: } \\
\hline Cryoeurolysis (CRYO) & & & $x$ & & & & & & \\
\hline Sham treatment (SHAM) & & & $x$ & & & & & & \\
\hline GLA:D (SHAM \& CRYO) & & & & $x$ & $x$ & & & & \\
\hline \multicolumn{10}{|l|}{ Assessments: } \\
\hline Pain & & & $x$ & $x$ & & $x$ & $x$ & $x$ & $x$ \\
\hline Functional performance & & & $x$ & $x$ & & $x$ & $x$ & $x$ & $x$ \\
\hline KOOS & & & $x$ & $x$ & & $x$ & $x$ & $x$ & $x$ \\
\hline EQ5D & & & $x$ & $x$ & & $x$ & $x$ & $x$ & $x$ \\
\hline Adverse effects & & & $x$ & $x$ & & $x$ & $x$ & $x$ & $x$ \\
\hline Analgesics & & & $x$ & $x$ & & $x$ & $x$ & $x$ & $x$ \\
\hline
\end{tabular}

$w$ Weeks, na Not applicable (GLA:D start time may vary) 


\section{Data monitoring}

The current project will not require a formal data monitoring committee. This decision is based on the minimal known risks associated with the intervention and little expected disturbance to the clinical equipoise. Any adverse effects will be recorded and reported according to the guidelines to the Regional Committee on Health Research Ethics for Southern Denmark within 7 days. Once a year the lead investigator will report all expected and unexpected adverse effects that have occurred during that period, with an evaluation of patient safety.

The leading investigators will review the trial processes and data continuously. In addition, a status report will be sent for approval to the Danish Health Authority each year.

\section{Data management}

All data will be kept electronically and filed according to a participant code. Data entry will always be handled by the same investigator, who will use unambiguous and standard terminology based on predefined study forms. Complete back-up of all data will be performed regularly and stored on OPEN's servers in the Region of Southern Denmark, using Research Electronic Data Capture (REDCap). REDCap store data via an encrypted connection with restricted access and fulfil the demands for data security. Data will be stored for a duration of 10 years. The project will be reported to the Danish Data Protection Agency and will be handled according to the regulations of the Act on Processing of Personal Data. The full data set will be available to the lead investigators only.

\section{Ethics}

The study is conducted according to the declaration of Helsinki and is approved by The Regional Committees on Health Research Ethics for Southern Denmark (S20180089) and registered in ClinicalTrials.gov (NCT03774121). Given the study design, eligibility criteria and safety measures, the current project does not include special risks for the participating patients. The majority of risks does not require medical attention and serious adverse effects as a result of local anesthetics and cryoneurolysis are very rare. In contrast cryoneurolysis could potentially provide an effective, safe and minimally invasive option to treat pain in OA patients.

The inclusion of a sham group is necessary to test the hypothesis because of the large reported placebo effects in invasive interventions [49]. In addition, the associated risks are minimal and the nature of misleading, in regards to the administration of the sham trial, will be adequately disclosed and accepted by the patient during the informed consent process. The sham group is implemented according to current standards [50].

\section{Discussion}

\section{Impact and significance of the study}

Current pain-relieving therapies show small to moderate effects and a significant portion of patients who receive surgery (TKA) report unchanged pain intensity levels post-surgery. This indicates that there is a need for additional therapies that could supplement or improve the existing therapies. A significant difference in the change in pain intensity between sham and intervention post cryoneurolysis treatment would indicate that cryoneurolysis is an effective non-pharmacological therapeutic option to treat pain in OA patients. Outcome measures include both pain, social and psychological factors and functional capacity, which allows for a thorough understanding of treatment effects. The novel addition of GLA:D in combination with cryoneurolysis will elucidate whether feasibility and effectiveness of exercise increase if pain is attenuated and if the potential pain-relieving effects of cryoneurolysis can be extended. The potential benefits include increased functional capacity and quality of life as a result of significant pain relief and improved benefits of physical exercise, ultimately postponing or making surgical intervention unnecessary. This could have a significant impact on patients' lives as well as significant socioeconomic consequences and could change the clinical landscape in the treatment of OA. Finally, an important perspective, is the application of cryoneurolysis in other areas - such as managing pain, related to surgery, to improve rehabilitation.

If the results indicate that cryoneurolysis does not elicit additional pain relief when compared to sham, it might indicate that cryoneurolysis treatment is not suitable for the treatment of OA related pain or at least not in the way it is applied in the current study.

Importantly, the freezing protocol in this study results in reversible nerve damage, allowing for the reinnervation of the sensory receptors over time $[15,16]$. It also means that pain sensation might return over time [17, $26,51,52]$. To maintain long-term pain-relieving effects, this study is the first to combine cryoneurolysis with a standardized education and exercise program. The analgesic effects of exercise are well established, however, the effects remain moderate and difficult to maintain over time [29]. Cryoneurolysis of the genicular nerves allows for potential long-term pain relief (> 3 months), without affecting motor control, which could help exercise feasibility and effectiveness - providing long-term benefits of both cryoneurolysis and exercise [34]. In the current study, patients are referred to GLA:D by their general practitioner and is performed independently of the study at specialized physiotherapists, post cryoneurolysis treatment. Differences in practice and starting time might occur between patients depending on the physiotherapist and some may choose to perform parts of their 


\section{Appendix}

Table 2 WHO data registration items

\begin{tabular}{|c|c|}
\hline Data category & Information \\
\hline Protocol version & Version 1 - 21 January 2021 \\
\hline Primary Registry & Clinicaltrial.gov \\
\hline Date of Registration & $12-03-2018$ \\
\hline Sources of Monetary & Danish Health Authority, Danish Rheumatism Association \\
\hline Primary Sponsor & Department of Neurology, Hospital South West Jutland, Esbjerg Denmark \\
\hline Secondary Sponsor & Research Unit of Health Sciences, Hospital South West Jutland, Esbjerg Denmark \\
\hline Contact for Public Queries & Niels-Peter Broechner Nygaard \\
\hline $\begin{array}{l}\text { Contact for Scientific } \\
\text { Queries }\end{array}$ & Niels-Peter Broechner Nygaard \\
\hline Country of Recruitment & Denmark \\
\hline Health Condition & Osteoarthritis (DM17) \\
\hline \multirow[t]{3}{*}{ Interventions } & Cryoneurolysis \\
\hline & Sham \\
\hline & GLA:D \\
\hline \multirow[t]{2}{*}{$\begin{array}{l}\text { Key inclusion and exclusion } \\
\text { criteria }\end{array}$} & $\begin{array}{l}\text { Inclusion: Referred to GLA:D, Chronic pain }>6 \text { months, Pain intensity } \geq 40 \mathrm{~mm} \text { on the NRS pain scale, OA confirmed by } \\
\text { radiography, } 50 \% \text { reduction of pain after diagnostic nerve block }\end{array}$ \\
\hline & $\begin{array}{l}\text { Exclusion: Systemic inflammatory disease, In treatment for other pain conditions, uncontrolled serious disease (cancer, } \\
\text { diabetes, etc.) }\end{array}$ \\
\hline \multirow[t]{3}{*}{ Study type } & Interventional \\
\hline & Allocation; Randomized controlled; Parallel assignment; Single-blinded (patients and data-manager) \\
\hline & Primary purpose; Safety and effectiveness \\
\hline Date if first enrollment & 26-06-20,189 \\
\hline Sample size & $n=94$ \\
\hline Recruitment status & Recruiting \\
\hline Primary outcome & Change in Pain intensity score (NRS) after cryoneurolysis, compared between intervention and sham \\
\hline \multirow[t]{3}{*}{ Key secondary outcomes } & Functional performance \\
\hline & Quality of Life \\
\hline & Adverse effects \\
\hline
\end{tabular}

training at home. These factors might confound the outcome of the exercise program but is consistent with clinical practice. The primary investigator facilitates GLA:D participation.

\section{Strength and limitations}

This study is a blinded randomized controlled sham trial that includes long-term follow ups allowing for an evaluation of the long-term effects of treatment. The sham intervention includes the same procedures as in cryoneurolysis treatment, emulating both physical marks and sensory experiences such as sound and vision. Despite of this, some performance bias cannot be excluded, and some attrition bias might occur, due to patients seeking treatment elsewhere - expectedly from the sham group. The inclusion of a sham group is necessary to test the hypothesis because of the large reported placebo effects in OA trials [49]. Treatment with cryoneurolysis is still in its early stage and further studies are needed to determine methodological strategies optimizing its potential therapeutic effects. To achieve long-term pain relief, significant degeneration of the sensory nerves is required. The extent of nerve damage depends on several factors including temperature, contact area, freezing rate, exposure time, thawing strategy and cell type. The current study applies a conservative freezing protocol, that is relatively short $\sim 3 \mathrm{~min}$, a single freezing cycle and not at full effect (slower freezing). This might reduce potential risks associated with the procedure but might also attenuate treatment effects. Other studies use up to several cycles and freezing periods up to $10 \mathrm{~min}$, which might induce pain relief for a longer duration due to increased nerve degeneration. These studies, however, tend to focus more on tissue destruction rather than degeneration. Further studies are needed to determine optimal freezing protocols relative to different use cases. 
The target nerves, the identification of the target nerves and placement of the cryo probe is equally as important and might have a significant impact on the results. The temperature gradient away from the probe increases drastically $[4,7]$. The typical isotherm with the needle (IceSeed 1.5, Galil Medical) used in this study has a temperature of $-40{ }^{\circ} \mathrm{C}$ in an area equivalent to $11 \times 20 \mathrm{~mm}$. Already at $20 \times 27 \mathrm{~mm}$ the temperature has risen to $-20^{\circ} \mathrm{C}$ and at $33 \times 38 \mathrm{~mm}$ the temperature is up to $0{ }^{\circ} \mathrm{C}[8]$. Thus, to attain temperatures necessary for a relevant impact on sensory nerves for long-term pain relief $\left[-20^{\circ} \mathrm{C}-100^{\circ} \mathrm{C}\right]$, probe placement relative to the nerve is crucial. The current study uses a combination of anatomical landmarks, electrical nerve stimulation and ultrasound to accurately determine nerve locations and to account for variation in surrounding anatomical structures. This both increase precision and safety of the procedure. Nevertheless, it is in some cases difficult to identify and differentiate nerve structures with ultrasound which might cause variable results. The procedure is in this study performed by the same surgeon throughout, trained to identify neural structures and to perform cryoneurolysis. In addition, local anesthesia is used locally only at the injection site, $4-6 \mathrm{~cm}$ from the 'target' nerve. This allows for continuous feedback on the effect of treatment.

\section{Dissemination of results}

The obtained results will be made publicly available within 1 year after the end of the project. This will include publication of the obtained results in international scientific peer-reviewed journals - adhering to the recommendations of the Vancouver convention. Published papers will have open access to ensure a broad distribution and the results will be presented at international scientific conferences. The results will also be presented for the general public and distributed across public platforms (e.g. regional and national press, internet sites, etc.) if copyright allows.

\section{Acknowledgements}

Not applicable.

\section{Authors' contributions}

NPBN: Major contributor to the conceptualization of the research idea and protocol. Primary contributor to the manuscript. Performs the acquisition and analysis of the results. Study management. CKJ; Major contributor to the conceptualization of the research idea and protocol. Performs the surgical intervention. Study management. HBV; Contributor to the conceptualization of the research idea and protocol. Manages patient-reported outcome database. NW; Contributor to the conceptualization of the research idea and protocol. Inclusion and diagnostics of patients. MBE; Contributor to the conceptualization of the research idea and protocol. Technical contributor to the surgical intervention and for the evaluation of pain. BG; Major contributor to the conceptualization of the research idea and protocol. Major contributor to the manuscript. Study management. All authors contributed to, read and approved the final manuscript.

\section{Funding}

The study was funded by the Danish Rheumatism Association and the Danish Health Authority. The funding sources does not have a role in the design of the study and collection, analysis and interpretation of data and in writing the manuscript. External peer-review took place during the funding process.

\section{Availability of data and materials}

Not applicable.

\section{Ethics approval and consent to participate}

The study is conducted according to the declaration of Helsinki and is approved by The Regional Committees on Health Research Ethics for Southern Denmark (S-20180089)

\section{Consent for publication}

Not applicable.

\section{Competing interests}

The authors declare that they have no competing interests.

\section{Author details}

${ }^{1}$ Research Unit of Health Science, Hospital of South West Jutland, University Hospital of Southern Denmark, Esbjerg, Denmark. ${ }^{2}$ Department of Regional Health Research, University of Southern Denmark, Odense, Denmark. ${ }^{3}$ Department of Neurology, Hospital South West Jutland, University Hospital of Southern Denmark, Esbjerg, Denmark. ${ }^{4}$ Pain Research Group, Odense University Hospital, Odense, Denmark. ${ }^{5}$ Department of Clinical Research, Faculty of Health Sciences, University of Southern Denmark, Odense, Denmark. ${ }^{6}$ Department of Orthopedics, Hospital South West Jutland, University Hospital of Southern Denmark, Esbjerg, Denmark.

Received: 21 January 2021 Accepted: 19 February 2021 Published online: 26 February 2021

\section{References}

1. O'Brien T, Breivik H. The impact of chronic pain-European patients' perspective over 12 months; 2012. p. 23-9.

2. Hootman JM, Helmick CG. Projections of US prevalence of arthritis and associated activity limitations. Arthritis Rheum. 2006;54(1):226-9.

3. Vos T, Flaxman AD, Naghavi M, Lozano R, Michaud C, Ezzati M, et al. Years lived with disability (YLDs) for 1160 sequelae of 289 diseases and injuries 1990-2010: a systematic analysis for the global burden of disease study 2010. Lancet. 2012;380(9859):2163-96.

4. March LM, Bachmeier CJ. Economics of osteoarthritis: a global perspective. Baillieres Clin Rheumatol. 1997;11(4):817-34.

5. Heidari B. Knee osteoarthritis prevalence, risk factors, pathogenesis and features: part I. Caspian J Intern Med. 2011;2(2):205-12.

6. Crawford DC, Miller LE, Block JE. Conservative management of symptomatic knee osteoarthritis: a flawed strategy? Orthop Rev (Pavia). 2013;5(1):e2.

7. Carr AJ, Robertsson O, Graves S, Price AJ, Arden NK, Judge A, et al. Knee replacement. Lancet. 2012;379(9823):1331-40.

8. Lewis GN, Rice DA, McNair PJ, Kluger M. Predictors of persistent pain after total knee arthroplasty: a systematic review and meta-analysis. $\mathrm{Br} J$ Anaesth. 2015;114(4):551-61.

9. Puolakka PA, Rorarius MG, Roviola M, Puolakka TJ, Nordhausen K, Lindgren L. Persistent pain following knee arthroplasty. Eur J Anaesthesiol. 2010;27(5): 455-60.

10. Liu SS, Buvanendran A, Rathmell JP, Sawhney M, Bae JJ, Moric M, et al. A cross-sectional survey on prevalence and risk factors for persistent postsurgical pain 1 year after total hip and knee replacement. Reg Anesth Pain Med. 2012:37(4):415-22.

11. McAlindon TE, Bannuru RR, Sullivan MC, Arden NK, Berenbaum F, BiermaZeinstra SM, et al. OARSI guidelines for the non-surgical management of knee osteoarthritis. Osteoarthr Cartil. 2014;22(3):363-88.

12. Basedow M, Esterman A. Assessing appropriateness of osteoarthritis care using quality indicators: a systematic review. J Eval Clin Pract. 2015;21(5): 782-9.

13. Sunderland S. A classification of peripheral nerve injuries producing loss of function. Brain. 1951;74(4):491-516. 
14. Iffeld BM, Preciado J, Trescot AM. Novel cryoneurolysis device for the treatment of sensory and motor peripheral nerves. Expert Rev Med Devices. 2016;13(8):713-25

15. Zhou L, Kambin P, Casey KF, Bonner FJ, O'Brien E, Shao Z, et al. Mechanism research of cryoanalgesia. Neurol Res. 1995;17(4):307-11.

16. Kerns JM, Braverman B, Mathew A, Lucchinetti C, Ivankovich AD. A comparison of cryoprobe and crush lesions in the rat sciatic nerve. Pain. 1991;47(1):31-9.

17. Birkenmaier C, Veihelmann A, Trouillier H, Hausdorf J, Devens C, Wegener B, et al. Percutaneous cryodenervation of lumbar facet joints: a prospective clinical trial. Int Orthop. 2007;31(4):525-30.

18. Allen BH, Fallat LM, Schwartz SM. Cryosurgery: an innovative technique for the treatment of plantar fasciitis. J Foot Ankle Surg. 2007:46(2):75-9.

19. Kim CH, Hu W, Gao J, Dragan K, Whealton T, Julian C. Cryoablation for the treatment of occipital neuralgia. Pain Physician. 2015;18(3):E363-8.

20. Moore W, Kolnick D, Tan J, YU HS. CT guided percutaneous cryoneurolysis for post thoracotomy pain syndrome: early experience and effectiveness. Acad Radiol. 2010;17(5):603-6.

21. Cazzato RL, Garnon J, Ramamurthy N, Tsoumakidou G, Caudrelier J, Thenint MA, et al. Percutaneous MR-guided cryoablation of Morton's neuroma: rationale and technical details after the first 20 patients. Cardiovasc Intervent Radiol. 2016;39(10):1491-8.

22. Franco CD, Buvanendran A, Petersohn JD, Menzies RD, Menzies LP. Innervation of the anterior capsule of the human knee: implications for radiofrequency ablation. Reg Anesth Pain Med. 2015;40(4):363-8.

23. Davis T, Loudermilk E, DePalma M, Hunter C, Lindley D, Patel N, et al. Prospective, multicenter, randomized, crossover clinical trial comparing the safety and effectiveness of cooled radiofrequency ablation with corticosteroid injection in the management of knee pain from osteoarthritis. Reg Anesth Pain Med. 2018;43(1):84-91.

24. Choi WJ, Hwang SJ, Song JG, Leem JG, Kang YU, Park PH, et al. Radiofrequency treatment relieves chronic knee osteoarthritis pain: a double-blind randomized controlled trial. Pain. 2011;152(3):481-7.

25. Zhou L, Craig J, Parekh N. Current concepts of neurolysis and clinical applications. J Analgesics. 2014;2:16-22.

26. Radnovich R, Scott D, Patel AT, Olson R, Dasa V, Segal N, et al. Cryoneurolysis to treat the pain and symptoms of knee osteoarthritis: a multicenter, randomized, double-blind, sham-controlled trial. Osteoarthr Cartil. 2017;25(8):1247-56.

27. Dasa V, Lensing G, Parsons M, Harris J, Volaufova J, Bliss R. Percutaneous freezing of sensory nerves prior to total knee arthroplasty. Knee. 2016;23(3): 523-8.

28. Trescot AM. Cryoanalgesia in interventional pain management. Pain Physician. 2003;6(3):345-60

29. Skou ST, Roos EM. Good Life with osteoArthritis in Denmark (GLA:D) evidence-based education and supervised neuromuscular exercise delivered by certified physiotherapists nationwide. BMC Musculoskelet Disord. 2017; 18(1):72.

30. Fransen M, McConnell S, Harmer AR, Van der Esch M, Simic M, Bennell KL. Exercise for osteoarthritis of the knee: a Cochrane systematic review. Br J Sports Med. 2015:49(24):1554-7.

31. Ageberg E, Link A, Roos EM. Feasibility of neuromuscular training in patients with severe hip or knee OA: the individualized goal-based NEMEX-TJR training program. BMC Musculoskelet Disord. 2010;11:126.

32. Kanavaki AM, Rushton A, Efstathiou N, Alrushud A, Klocke R, Abhishek A, et al. Barriers and facilitators of physical activity in knee and hip osteoarthritis: a systematic review of qualitative evidence. BMJ Open. 2017; 7(12):e017042

33. Gay C, Eschalier B, Levyckyj C, Bonnin A, Coudeyre E. Motivators for and barriers to physical activity in people with knee osteoarthritis: a qualitative study. Joint Bone Spine. 2018;85(4):481-6.

34. Stelzer W, Stelzer V, Stelzer D, Braune M, Duller C. Influence of BMI, gender, and sports on pain decrease and medication usage after facet-medial branch neurotomy or SI joint lateral branch cooled RF-neurotomy in case of low back pain: original research in the Austrian population. J Pain Res. 2017; 10:183-90.

35. Hu E, Preciado J, Dasa V, Mussell J. Development and validation of a new method for locating patella sensory nerves for the treatment of inferior and superior knee pain. J Exp Orthop. 2015;2(1):16
36. Le Corroller T, Lagier A, Pirro N, Champsaur P. Anatomical study of the infrapatellar branch of the saphenous nerve using ultrasonography. Muscle Nerve. 2011:44(1):50-4

37. Hawker GA, Mian S, Kendzerska T, French M. Measures of adult pain: visual analog scale for pain (VAS pain), numeric rating scale for pain (NRS pain), McGill pain questionnaire (MPQ), short-form McGill pain questionnaire (SF$M P Q)$, chronic pain grade scale (CPGS), short form-36 bodily pain scale (SF$36 \mathrm{BPS})$, and measure of intermittent and constant osteoarthritis pain (ICOAP). Arthritis Care Res. 2011;63(Suppl 11):S240-52.

38. Dobson F, Hinman RS, Roos EM, Abbott JH, Stratford P, Davis AM, et al. OARSI recommended performance-based tests to assess physical function in people diagnosed with hip or knee osteoarthritis. Osteoarthr Cartil. 2013; 21(8):1042-52.

39. Jones CJ, Rikli RE, Beam WC. A 30-s chair-stand test as a measure of lower body strength in community-residing older adults. Res Q Exerc Sport. 1999; 70(2):113-9.

40. Wright AA, Cook CE, Baxter GD, Dockerty JD, Abbott JH. A comparison of 3 methodological approaches to defining major clinically important improvement of 4 performance measures in patients with hip osteoarthritis. J Orthop Sports Phys Ther. 2011:41(5):319-27.

41. Kennedy DM, Stratford PW, Wessel J, Gollish JD, Penney D. Assessing stability and change of four performance measures: a longitudinal study evaluating outcome following total hip and knee arthroplasty. BMC Musculoskelet Disord. 2005;6:3.

42. Roos EM, Toksvig-Larsen S. Knee injury and osteoarthritis outcome score (KOOS) - validation and comparison to the WOMAC in total knee replacement. Health Qual Life Outcomes. 2003;1:17.

43. Herdman M, Gudex C, Lloyd A, Janssen M, Kind P, Parkin D, et al. Development and preliminary testing of the new five-level version of EQ-5D (EQ-5D-5L). Qual Life Res. 2011;20(10):1727-36.

44. Sullivan MJL, Bishop SR, Pivik J. The pain catastrophizing scale: development and validation. Psychol Assess. 1995;7(4):524-32.

45. Kroenke K, Spitzer RL, Williams JB. The PHQ-9: validity of a brief depression severity measure. J Gen Intern Med. 2001;16(9):606-13.

46. Löwe B, Decker O, Müller S, Brähler E, Schellberg D, Herzog W, et al. Validation and standardization of the generalized anxiety disorder screener (GAD-7) in the general population. Med Care. 2008;46(3):266-74.

47. Tubach F, Ravaud P, Baron G, Falissard B, Logeart I, Bellamy N, et al. Evaluation of clinically relevant changes in patient reported outcomes in knee and hip osteoarthritis: the minimal clinically important improvement. Ann Rheum Dis. 2005;64(1):29-33.

48. Sun HH, Zhuang SY, Hong X, Xie XH, Zhu L, Wu XT. The efficacy and safety of using cooled radiofrequency in treating chronic sacroiliac joint pain: a PRISMA-compliant meta-analysis. Medicine (Baltimore). 2018:97(6):e9809.

49. de Campos GC. Placebo effect in osteoarthritis: why not use it to our advantage? World J Orthop. 2015;6(5):416-20

50. Horng S, Miller FG. Ethical framework for the use of sham procedures in clinical trials. Crit Care Med. 2003:31(3 Suppl):S126-30.

51. Moorjani N, Zhao F, Tian Y, Liang C, Kaluba J, Maiwand MO. Effects of cryoanalgesia on post-thoracotomy pain and on the structure of intercostal nerves: a human prospective randomized trial and a histological study. Eur J Cardiothorac Surg. 2001;20(3):502-7.

52. Wang JK. Cryoanalgesia for painful peripheral nerve lesions. Pain. 1985;22(2): $191-4$.

\section{Publisher's Note}

Springer Nature remains neutral with regard to jurisdictional claims in published maps and institutional affiliations. 